\title{
Diagnosis of Churg-Strauss Syndrome Presented With Neuroendocrine Carcinoma: A Case Report
}

\author{
Dayun Park, MD ${ }^{1}$, Ho Jun Lee, $\mathrm{MD}^{1}$, Kwang Hoon Lee, $\mathrm{MD}^{2}$, Bum Sun Kwon, MD ${ }^{1}$, \\ Jin-Woo Park, MD', Ki Yeun Nam, MD' ${ }^{1}$ Kyoung Hwan Lee, $\mathrm{MD}^{1}$ \\ Departments of ${ }^{1}$ Physical Medicine and Rehabilitation and ${ }^{2}$ Internal Medicine, \\ Dongguk University College of Medicine, Seoul, Korea
}

\begin{abstract}
Churg-Strauss syndrome (CSS) is a rare systemic vasculitis that affect small and medium-sized blood vessels and is accompanied by asthma, eosinophilia, and peripheral neuropathy. This report describes a case of a 52-yearold man who had a history of sinusitis, asthma, and thymus cancer and who had complained of bilateral lower extremity paresthesia and weakness for a month. Peripheral neuropathy was detected by electrodiagnostic studies. Resection of a mediastinal mass, which was diagnosed as thymic neuroendocrine carcinoma, was performed five months before his visit. After thymectomy, peripheral blood tests revealed a gradual increase in eosinophils. Two months after surgery, he was admitted to the hospital for dyspnea, and nodules of focal consolidation were found in his chest X-ray. One month later, pyoderma occurred in the right shin, and the skin biopsy showed extravascular eosinophilic infiltration. He was diagnosed with CSS after thymectomy, and we report a very rare case of CSS presented with thymic neuroendocrine carcinoma.
\end{abstract}

Keywords Churg-Strauss syndrome, Thymic neuroendocrine carcinoma, Polyneuropathy

\section{INTRODUCTION}

Churg-Strauss syndrome (CSS) was first described in 1951 by Churg and Strauss [1], who reported it as being associated with clinical aspects of asthma, eosinophilia, vasculitis contracted in various organs, skin lesions, and neurological symptoms.

A few cases of vasculitis associated with malignancies have been reported [2-5]. However, there has been only one reported case of thymic neuroendocrine carcinoma accompanied by vasculitis that was diagnosed as CSS [6]. We report a new case of CSS with thymic neuroendocrine carcinoma, vasculitis based on peripheral neuropathy, eosinophilia, pulmonary infiltration, and a history of asthma and paranasal sinusitis. This case had an unusual and previously unreported clinical presentation of CSS

Received May 16, 2016; Accepted August 29, 2016

Corresponding author: Ho Jun Lee

Department of Physical Medicine and Rehabilitation, Dongguk University Ilsan Hospital, Dongguk University College of Medicine, 27 Dongguk-ro, Ilsandong-gu, Goyang 10326, Korea. Tel: +82-31-961-7460, Fax: +82-31-961-7488, E-mail: hjrhee1@gmail.com

ORCID: Dayun Park (http://orcid.org/0000-0003-1685-9023); Ho Jun Lee (http://orcid.org/0000-0002-1997-2593); Kwang Hoon Lee (http://orcid. org/0000-0002-9506-9072); Bum Sun Kwon (http://orcid.org/0000-0001-7755-435X); Jin-Woo Park (http://orcid.org/0000-0003-4989-2575); Ki Yeun Nam (http://orcid.org/0000-0001-6932-6541); Kyoung Hwan Lee (http://orcid.org/0000-0002-8720-3315).

@ This is an open-access article distributed under the terms of the Creative Commons Attribution Non-Commercial License (http://creativecommons.org/ licenses/by-nc/4.0) which permits unrestricted noncommercial use, distribution, and reproduction in any medium, provided the original work is properly cited. Copyright $\odot 2017$ by Korean Academy of Rehabilitation Medicine 
along with peripheral neuropathy and worsening CSS symptoms after thymectomy.

\section{CASE REPORT}

A 52-year-old male patient visited the hospital with paresthesia and motor weakness in both legs that lasted for a month. Neurologic deficits were found as follows. A manual muscle test revealed only distal weakness, that is, poor grades in dorsiflexors, plantarflexors of both ankles and flexors, and extensors of both big toes. A sensory examination revealed a decrease in light touches in both feet. The deep tendon reflexes of all extremities were normal. There was no deformity or tenderness of either foot. Though he had walked without difficulty until 2 months before the visit, he had gait disturbance with foot drop on both legs.

He had been previously diagnosed with asthma and sinusitis (Fig. 1). He had had an anterior mediastinal mass removed 5 months before visiting the hospital because of leg weakness. The pathologic diagnosis of the mass was as an atypical carcinoid of neuroendocrine carcinoma. Immunohistochemistry showed that the CD56(+) and Ki$67(+)$ index was approximately $10 \%$ (Fig. 2A). One month after the surgery, radiotherapy was conducted 30 times in $60 \mathrm{~Gy}$ for 40 days. Ten days after completion of the 40-day radiation therapy, the patient was admitted the hospital, chiefly complaining of dyspnea. The chest X-ray revealed poorly defined nodules of focal consolidation in the right upper and lower lung lobes (Fig. 3). The symptom of dyspnea was alleviated after 2 weeks of treatment and the patient was discharged. Three weeks later, tingling sensations and weakness in both feet and both ankles occurred and lasted for a month. He was admitted to our department for further evaluation. During hospitalization, pyoderma was found in the right shin.

The nerve conduction study showed decreased amplitude and delayed latency of the compound motor action

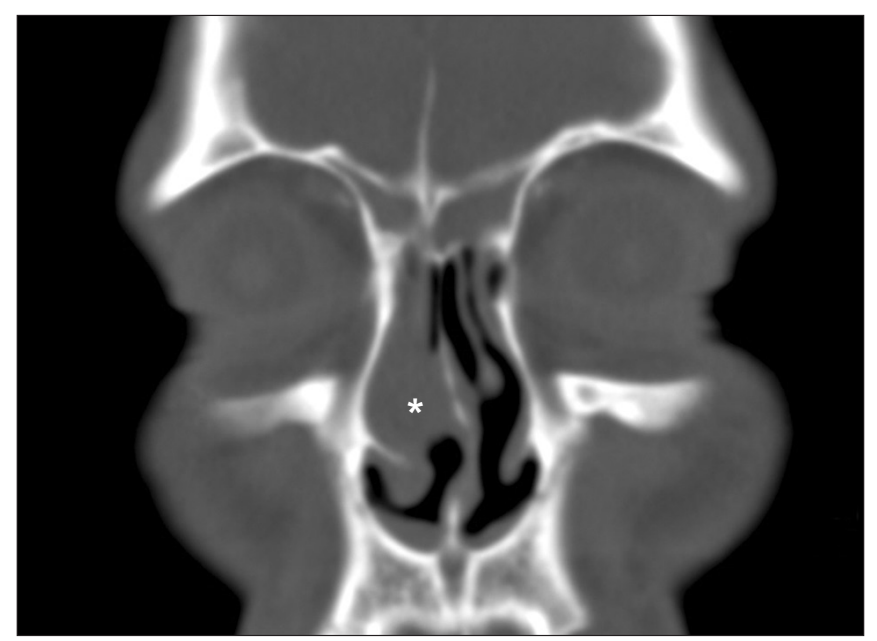

Fig. 1. Paranasal sinus computed tomography in the coronal section showed sinusitis and mucosal thickening (asterisk).
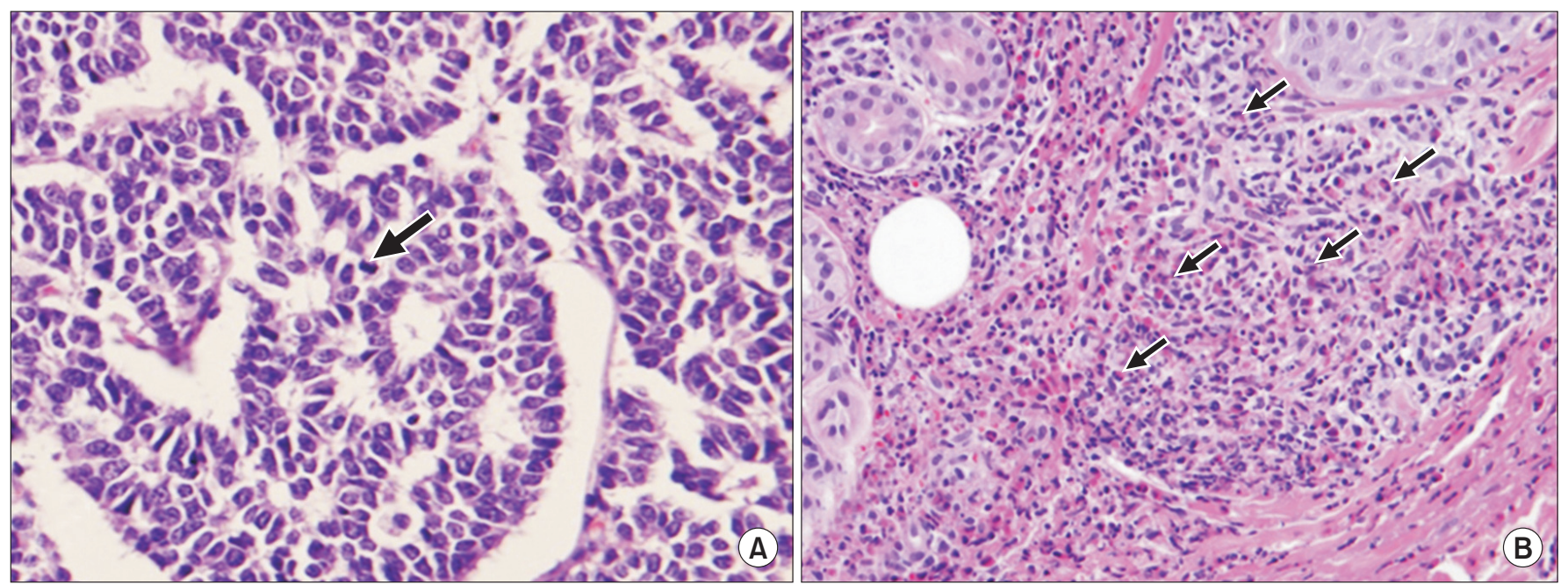

Fig. 2. Mediastinal mass excisional biopsy (A) showed a composition of medium-sized polygonal cells with mild pleomorphism. Atypical mitosis is noted (black arrow) (H\&E, $\times 720)$. Skin punch biopsy of right shin (B) showed extravascular eosinophil infiltration $(\mathrm{H} \& \mathrm{E}, \times 400)$. 
potential (CMAP) and sensory nerve action potential (SNAP) in the right median nerve, no response of the CMAP and SNAP in both common peroneal and right tibial nerves, decreased amplitude of the CMAP in the left tibial nerve, and no response of the SNAP in both su-

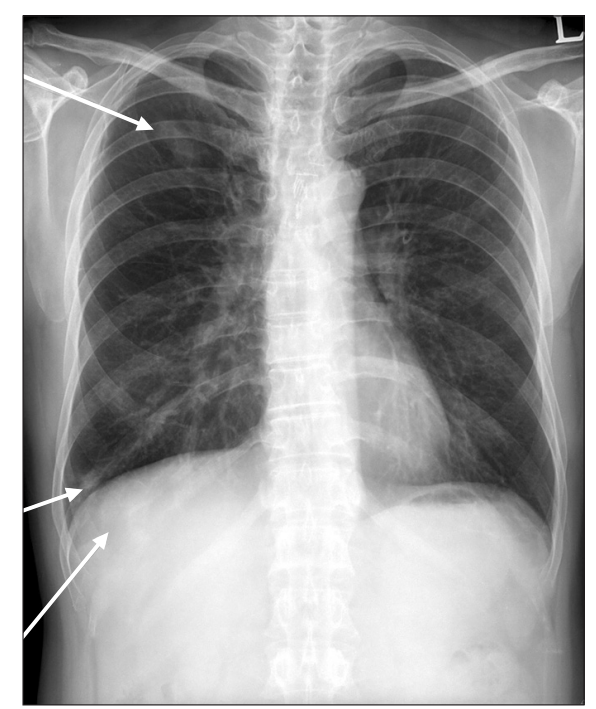

Fig. 3. Chest X-ray showed poorly defined nodules of focal consolidation in the right upper and lower lobes. perficial peroneal and sural nerves (Table 1). The needle electromyography (EMG) showed active denervation in the right anterior tibialis and right abductor pollicis brevis with long duration polyphasic motor unit action potential (MUAP) and reduced recruitment (Table 2). An asymmetric pattern of involvement was revealed in CMAP, SNAP, and needle EMG. The mononeuropathy multiplex was diagnosed in the electrodiagnostic studies. The biopsy of pyoderma containing blood vessels showed extravascular eosinophils, which was compatible with vasculitis (Fig. 2B).

The results of the laboratory tests were as follows. Antineutrophil cytoplasmic antibody (ANCA) negative, serum adrenocorticotropic hormone (ACTH), cortisol (at 8 AM) and urine 5-hydroxyindoleacetic acid (HIAA) were all within normal limits. The number of peripheral blood eosinophils was higher than normal (22\%) before thymectomy. After thymectomy, the number of peripheral blood eosinophils began to gradually rise. The eosinophils and total IgE levels were measured as $45.2 \%$ and $1,163 \mathrm{kU} / \mathrm{L}$, respectively, higher than the normal values.

The patient was diagnosed as CSS according to the American College of Rheumatology (ACR) 1990 criteria [7], because the past history and the present clinical

Table 1. Nerve conduction studies

\begin{tabular}{|c|c|c|c|c|c|c|c|c|}
\hline & \multirow{2}{*}{ Stimulation site } & \multirow{2}{*}{ Recording site } & \multicolumn{2}{|c|}{ Amplitude } & \multicolumn{2}{|c|}{ Latency (ms) } & \multicolumn{2}{|c|}{$\mathrm{CV}(\mathrm{m} / \mathrm{s})$} \\
\hline & & & Right & Left & Right & Left & Right & Left \\
\hline \multicolumn{9}{|c|}{ Motor nerve } \\
\hline \multirow[t]{2}{*}{ Median } & Wrist & APB & 1.3 & - & 4.50 & - & - & - \\
\hline & Elbow & APB & 1.0 & - & 9.10 & - & 50 & - \\
\hline \multirow[t]{2}{*}{ Ulnar } & Wrist & $\mathrm{ADQ}$ & 7.4 & - & 2.95 & - & - & - \\
\hline & Elbow & $\mathrm{ADQ}$ & 6.6 & - & 7.05 & - & 56.1 & - \\
\hline \multirow[t]{2}{*}{ Peroneal } & Ankle & EDB & NE & $\mathrm{NE}$ & NE & $\mathrm{NE}$ & - & - \\
\hline & Fibular head & EDB & $\mathrm{NE}$ & $\mathrm{NE}$ & $\mathrm{NE}$ & $\mathrm{NE}$ & $\mathrm{NE}$ & $\mathrm{NE}$ \\
\hline \multirow[t]{2}{*}{ Tibial } & Ankle & $\mathrm{AH}$ & NE & 2.3 & $\mathrm{NE}$ & 3.80 & - & - \\
\hline & Popliteal fossa & $\mathrm{AH}$ & $\mathrm{NE}$ & 1.9 & $\mathrm{NE}$ & 2.90 & NE & 42.9 \\
\hline \multicolumn{9}{|c|}{ Sensory nerve } \\
\hline Median & Wrist & Second finger & 2.1 & - & 2.90 & - & - & - \\
\hline Ulnar & Wrist & Fifth finger & 16.6 & - & 2.60 & - & - & - \\
\hline Peroneal & Lateral leg & Foot & $\mathrm{NE}$ & $\mathrm{NE}$ & $\mathrm{NE}$ & $\mathrm{NE}$ & - & - \\
\hline Sural & Calf & Lateral malleolus & NE & NE & $\mathrm{NE}$ & $\mathrm{NE}$ & - & - \\
\hline
\end{tabular}

Amplitudes are measured in millivolt ( $\mathrm{mV}$, motor) and in microvolt ( $\mu \mathrm{V}$, sensory). All motor and sensory latencies are onset latencies. Left median, ulnar, and radial nerve conduction studies were not performed.

$\mathrm{CV}$, conduction velocity; $\mathrm{APB}$, Abductor pollicis brevis; $\mathrm{ADQ}$, Abductor digiti quinti; $\mathrm{EDB}$, extensor digitorum brevis; AH, abductor hallucis; NE, not evoked. 
Table 2. Needle electromyography studies

\begin{tabular}{lccccccccc}
\hline & \multicolumn{3}{c}{ Spontaneous activity } & & \multicolumn{3}{c}{ Motor unit action potential } \\
\cline { 2 - 3 } & IA & Fib & PSW & & $\begin{array}{c}\text { Amplitude } \\
(\mu \mathbf{V})\end{array}$ & $\begin{array}{c}\text { Duration } \\
(\mathbf{m s})\end{array}$ & PPP & $\begin{array}{c}\text { Recruitment } \\
\text { pattern }\end{array}$ \\
\hline Right first dorsal interossei & $\mathrm{N}$ & $\mathrm{N}$ & $\mathrm{N}$ & & $\mathrm{N}$ & $\mathrm{N}$ & $\mathrm{N}$ & Complete \\
Right abductor pollicis brevis & $\mathrm{N}$ & $1+$ & $3+$ & & + & + & Reduced \\
Right biceps brachii & $\mathrm{N}$ & $\mathrm{N}$ & $\mathrm{N}$ & & $\mathrm{N}$ & $\mathrm{N}$ & Complete \\
Right tibialis anterior & $\mathrm{N}$ & $1+$ & $3+$ & & $\mathrm{N}$ & + & + & Single \\
Right vastus medialis & $\mathrm{N}$ & $\mathrm{N}$ & $\mathrm{N}$ & $\mathrm{N}$ & $\mathrm{N}$ & $\mathrm{N}$ & Complete \\
\hline
\end{tabular}

IA, insertional activity; Fib, fibrillation; PSW, positive sharp wave; PPP, polyphasic pattern; N, normal.

manifestations included asthma, eosinophilia, pulmonary infiltration, peripheral neuropathy, and extravascular eosinophils. The patient was treated with oral corticosteroid (50 mg/day), received daily intervention of superficial and deep heat and electrical stimulation for pain relief, and relaxation of soft tissue and rehabilitation, including therapeutic exercise to improve flexibility, strengthening, and gait (twice a day, for a month). $\mathrm{He}$ displayed gradual improvement of eosinophilia, of the tingling sensation on both feet, and of weakness in both ankles up to the manual muscle test grade 3 .

\section{DISCUSSION}

Autoimmune disease, such as vasculitis, has been associated with malignancy. It is more frequent in hematologic malignancies than in solid tumors. The relationship between vasculitis and malignancy remains unclear. Vasculitis manifested cutaneous involvement, peripheral neuropathy, organ involvement, and joint syndrome. They were described as cutaneous leukocytoclastic vasculitis, Wegener's granulomatosis, microscopic polyangiitis, CSS, and polyarteritis nodosa [2-5]. Vasculitis accompanying the mediastinal carcinoma had been rarely reported, with only two cases of CSS among them $[3,4]$. There had been only one report of vasculitis associated with thymic neuroendocrine carcinoma like this case; in it, CSS and symptoms were alleviated after thymectomy [6]. However, in our case, eosinophils gradually increased, and symptoms of dyspnea and peripheral neuropathy began to appear after thymectomy, leading to the diagnosis of CSS. The temporal relationship of malignancy and vasculitis is variable. Vasculitis may occur before or after the onset of a cancer or coexist with it. ANCA, which generally appears positive in CSS, frequently appears negative in cancer-related vasculitis, as in our case [8].

Vasculitis accompanying a malignancy may be treated by corticosteroids, immunosuppressant drugs, or surgical procedures. Though recurrence might be possible even after surgical resection, most of the vasculitis improves if the malignancy is well treated [2-5]. However, autoimmune disease does not necessarily get better after treatment of an underlying malignancy, as is the case for the malignancy associated diseases of polymyositis and dermatomyositis [9]. Parambli et al. [10] reported the exacerbation of polyangiitis after thymectomy in a case of microscopic polyangiitis associated with thymoma.

In this case, there was no improvement of symptoms after thymectomy. The possibility of simultaneous occurrence of CSS and thymic neuroendocrine carcinoma could not be ruled out. Though the CSS symptom appeared after the surgery, there was a temporal relationship between the cancer and the outbreak of CSS. Even before surgery, the level of peripheral eosinophilia was $10 \%$ higher, and the patient had a history of asthma and paranasal sinusitis. Radiotherapy was additionally performed because of a positive margin after surgery, and we could not exclude the possibility that a minimal residual tumor might have affected the manifestation of CSS. Thus, we could not rule out the possibility that CSS had presented as a paraneoplastic syndrome. Although recurrence of the cancer has not been observed, continued follow-up is needed. If cancer recurs during followup, the symptoms of CSS may have predictive value. CSS rarely accompanies a solid tumor, and there is only one case report, in particular, where it has accompanied thymic neuroendocrine carcinoma.

By means of clinical symptoms, blood tests, and skin biopsy, we diagnosed the rare disease of CSS in a patient 
who was diagnosed with peripheral polyneuropathy by electrodiagnostic examination. The clinical manifestations of CSS might be different, depending on the timing and progress of the disease. If abnormalities are found in the patient for whom the cause of neuropathy is unclear, it is necessary to carefully look into the medical history of the patient, and consider the possibility of other autoimmune diseases like CSS as well.

\section{CONFLICT OF INTEREST}

No potential conflict of interest relevant to this article was reported.

\section{REFERENCES}

1. Churg J, Strauss L. Allergic granulomatosis, allergic angiitis, and periarteritis nodosa. Am J Pathol 1951;27: 277-301.

2. Fain O, Hamidou M, Cacoub P, Godeau B, Wechsler B, Paries J, et al. Vasculitides associated with malignancies: analysis of sixty patients. Arthritis Rheum 2007; 57:1473-80.

3. Solans-Laque R, Bosch-Gil JA, Perez-Bocanegra C, Selva-O'Callaghan A, Simeon-Aznar CP, VilardellTarres M. Paraneoplastic vasculitis in patients with solid tumors: report of 15 cases. J Rheumatol 2008;35:
294-304.

4. Hutson TE, Hoffman GS. Temporal concurrence of vasculitis and cancer: a report of 12 cases. Arthritis Care Res 2000;13:417-23.

5. Kurzrock R, Cohen PR. Vasculitis and cancer. Clin Dermatol 1993;11:175-87.

6. Liu T, Cai B, Feng R. Churg-Strauss syndrome presented as paraneoplastic syndrome with thymic neuroendocrine carcinoma: a case report. Rheumatol Int 2012;32:3683-5.

7. Masi AT, Hunder GG, Lie JT, Michel BA, Bloch DA, Arend WP, et al. The American College of Rheumatology 1990 criteria for the classification of Churg-Strauss syndrome (allergic granulomatosis and angiitis). Arthritis Rheum 1990;33:1094-100.

8. Racanelli V, Prete M, Minoia C, Favoino E, Perosa F. Rheumatic disorders as paraneoplastic syndromes. Autoimmun Rev 2008;7:352-8.

9. Andras C, Ponyi A, Constantin T, Csiki Z, Szekanecz E, Szodoray P, et al. Dermatomyositis and polymyositis associated with malignancy: a 21-year retrospective study. J Rheumatol 2008;35:438-44.

10. Parambil JG, Keogh KA, Fervenza FC, Ryu JH. Microscopic polyangiitis associated with thymoma, exacerbating after thymectomy. Am J Kidney Dis 2006;48: 827-31. 